\title{
Alphanumeric category effects: Evidence against the sufficiency of a localization explanation
}

\author{
BEN (C) FLETCHER \\ The Hatfield Polytechnic, Hatfield, Herts AL10 9AB, England
}

\begin{abstract}
The experiment utilized a serial choice reaction time (RT) paradigm in which only one alphanumeric stimulus was presented per trial, and the target set consisted of a single identified item. The categorical relationship between the target and nontarget items was varied as a property of blocks of trials. Target and nontarget RTs were smaller when the specified target item (e.g., the number 6) was categorically distinct from the nontargets (e.g., letters) than when it was from the same category (e.g., digits). The processing of catch-trial stimuli (items from the alternate category to the nontargets) and homographic category-ambiguous items was inhibited only in the former, between-category, condition. The results are contrasted with those obtained in visual search tasks. They suggest that a "locational-cue" explanation of alphanumeric category effects is inadequate.
\end{abstract}

The experiment reported here investigates the processes responsible for alphanumeric category effects and attempts to provide a clearer understanding of the role of identity and category information in the perception of letters and digits.

A central question in the existing literature is whether or not alphanumeric characters can be categorized before they are more specifically identified. Experimental evidence is contradictory.

Various kinds of effects have been taken as evidence that categorical information from a visual array can be extracted independently and prior to the explicit identification of the constituent display items:

(1) Using a tachistoscopic variant of the visual search technique developed by Neisser (1963), it has been found consistently that if target and field items are from the same alphanumeric category (withincategory condition), the function relating RT to the number of display items has a pronounced linear slope of about $35 \mathrm{msec} /$ item (Atkinson, Holmgren, \& Juola, 1969; Sternberg, 1967). If, on the other hand, the target and field items are from different categories (between-category condition), the function is much smaller, or even flat (Egeth, Atkinson, Gilmore, \& Marcus, 1973; Egeth, Jonides, \& Wall, 1972). The work of Jonides and Gleitman (1972, 1976; Gleitman \& Jonides, 1976, 1978), in which within- and between-category conditions can be directly compared, is the most widely cited and provides the most comprehensive investigation of the effects using this technique.

This work was done while the author was in receipt of a Medical Research Council grant at the Department of Experimental Psychology, Oxford University. My thanks are extended to P. M. A. Rabbitt for helpful advice and to an anonymous reviewer for perceptive comments.
(2) Search times through large arrays of items are faster if the field items are drawn from an alphanumeric category that is different from that of the target items (Brand, 1971; Ingling, 1972).

(3) Selective masking of target digits presented in the middle of a row of stimuli does not occur if the field characters are letters (Butler, 1975).

If categorization does precede identification, as a number of researchers have suggested (Brand, 1971; Deutsch, 1977; Ingling, 1972; Jonides \& Gleitman, 1976), two predictions should follow. First, categorydefined target search (e.g., any digit) should be faster than identity-defined search (e.g., "the digit 6") if the field items are drawn from the other category (e.g., letters). Second, naming the category of an item should take less time than specifying its identity.

Neither of these predictions has been confirmed. Dick (1971) reports that subjects could name visually presented alphanumeric characters some $150 \mathrm{msec}$ faster than they could name the category to which they belonged. Furthermore, both identification and categorization times increased by a constant amount when the number of alternative characters was varied, suggesting that an item's category was derived from its name. White $(1980,1977$, Experiments 1-4) consistently found that identification times were smaller than categorization RTs whether or not the target item was presented singly or in the context of seven other items, and even after nine practice sessions. White (1977) also found that identification and categorization times increased by a constant amount with the addition of context items. A comparison of "present" RTs for category-defined target search with identity-defined targets in Egeth, Jonides, and Wall (1972, compare Experiments 2 and 3) reveals that the former took some $80 \mathrm{msec}$ longer 
than the latter at all display sizes. Taylor (1978, Experiment 3) obtained the usual between/withincategory slope effects and found that at the larger display sizes, RTs were smaller after a category-specified search instruction (between-category condition) than after an identity-defined search instruction (withinsearch condition). However, from an analysis of projected intercepts, Taylor concluded that identification was a faster-terminating process than categorization. Both Brand (1971) and Sperling, Budiansky, Spivak, and Johnson (1971), on the other hand, reported that some unpracticed and many practiced subjects can search for an unspecified category-defined target as fast as they can for a specified target of that category if the field items are drawn from the other category. One explanation of these discrepant results, supported by the subjective reports from Brand's study (1971, p. 184), is that subjects adopted a category-level search strategy even when they were given the identity of the target. The equivalence of these search rates does not, of course, support the view that categorization precedes identification; none of the subjects were able to search faster for categorylevel targets than they were for identity-level targets.

How, then, are the slope-comparison results to be reconciled with the equally robust findings of faster explicit identification times?

A recent attempt to reconcile the apparent inconsistency has been to propose that the flatter slopes in the between-category conditions does not reflect that categorization is faster than identification, but the selective processing of a limited-capacity processor. Butler (1980), for example, suggested that performance in within-category conditions is constrained by stimulus localization factors, rather than by the relative speed of stimulus identification and categorization. The categorical distinction between the target and background items in the between-category condition "allows the localisation process to focus on the target item, thereby avoiding the need to locate nontarget stimuli" (Butler, 1980, p. 240). This "locational hypothesis" is supported by a number of findings. Gleitman and Jonides (1976) showed that the difference in RT between reporting a target's spatial location and naming the target was much smaller in the between- than in the withincategory condition. The facilitatory effect on RT of precuing target location was also smaller in the between-category condition. Butler (1980) briefly presented subjects with a 9-character array which included 1, 5, or 9 letters. The most common kind of errors, which distinguished between search conditions, were mislocations (reporting items adjacent to the target) rather than intrusions (items not presented). Butler proposes that his interpretation "is entirely consistent with all observations but avoids the paradox of assuming that category is determined prior to identity"' (p. 245).

The "locational hypothesis" is a particularly appealing explanation of the effects because it is consistent both with subjects' reported experiences and with experimental evidence that suggests that less information about each nontarget item is extracted from the array when the target and background items are categorically distinct. Taylor (1978), for example, concludes that in the between-category conditions "the best description of the data is that ... subjects behaved as though the non-targets were not even there" (p. 435). In his original work on visual search, Neisser (1963) offered an analogous explanation for rapid processing rates: the subject "does not 'see' the letters he passes; he 'sees' only a blur until the $z$ 'jumps out at him" " (p. 380). Gleitman and Jonides (1976) report that, in a subsequent recognition task, fewer nontargets were recalled in the between- than in the within-category condition.

The locational hypothesis does not provide an adequate framework in several important respects. Unless it is proposed that early categorization processes guide the locational processes, the difficulty remains of explaining why the category effects obtain. The hypothesis does not predict, for example, subjects' tendency to respond "present" in between-category conditions to a catch trial containing an item from the target category which was not one of the targets specified for that trial (Gleitman \& Jonides, 1976); it does not predict the apparent partial processing of target items. Furthermore, it would appear that the operation of location cuing is not an automatic consequence of the categorical distinction per se. Taylor (1978) presents evidence to show that in betweencategory conditions with multiple arrays, advance knowledge of the category of the category-discrepant item is necessary for facilitation to occur. Gleitman and Jonides (1978) have demonstrated that when the subject cannot form a set for the target items (i.e., when they are from different categories), the between/ within-category slope differences are eliminated.

The purpose of the experiment reported here was to investigate whether the localization facilitation in between-category conditions was necessary for category effects to obtain. The experiment involved subjects' doing a serial choice reaction time task with an array size of only a single item which always occurred in the center of the display. The between/ within-category variable was manipulated as a property of blocks of trials. Any category effects present cannot be logically attributed to location facilitating cues and must be interpreted in some other framework. It is also worth pointing out that, in multiple array studies, one cannot easily infer whether the processing facilitation in between-category con- 
ditions is due to faster target processing per se, faster nontarget rejections, or both of these factors. The serial CRT paradigm does permit such comparisons.

\section{METHOD}

\section{Subjects}

The subjects were nine right-handed students, aged 20-26 years, with normal or corrected-to-normal vision. Five were female and four were male. They were paid at an hourly rate.

\section{Design}

Each subject performed all experimental conditions described below in a single 60 -min session. The subjects were tested individually.

\begin{abstract}
Apparatus
A Digital Equipment Corporation (DEC) Linc-8 computer was programmed to provide the different versions of the two-choice serial self-paced RT tasks. The letters and digits were from the standard DEC $6 \times 4$ matrix character set, averaging $2.5 \mathrm{~mm}$ in width and $3 \mathrm{~mm}$ in height. They were observed at a distance of $50 \mathrm{~cm}$ through a viewing tube with a headrest. Each character appeared illuminated in green, in the center of a $20 \times 15 \mathrm{~cm}$ cathode ray tube display (coated with $P 11$ phosphor, which decays to $10 \%$ in $80 \mu \mathrm{sec}$ ). The two response keys, marked "TARGET" and "NO," were conveniently inset in a desk beneath where the subjects' two forefingers would be. As soon as either key was pressed, the current signal disappeared and the next item in the pseudorandom sequence appeared after a fixed response-stimulus interval of $300 \mathrm{msec}$. RT was timed to within $1 \mathrm{msec}$.
\end{abstract}

\section{Procedure and Conditions}

Each subject was given time to dark-adapt, during which the nature of the task was explained. At the beginning of each block or run of trials, the target character for that run was specified. The subjects were instructed to respond to each character by pressing the appropriate response key. They were told that most characters in a run would require a "no" response but that they should be careful not to miss any of the targets. There were two experimental conditions, each with two subconditions: (1) withincategory conditions, in which all the target and nontarget items within a block of trials were chosen from one category-letter targets and letter nontargets $(\mathrm{L} / \mathrm{L})$ and digit targets and digit nontargets (D/D); and (2) between-category conditions, in which the specified target item was categorically distinct from the nontarget items in the run-digit target with letter nontargets $(D / L)$ and letter target with digit nontargets (L/D).

The number of trials in a run varied for two reasons. First, the specified target item occurred 3,4 , or 5 times in a block of trials in order to maintain choice uncertainty throughout the run. Second, the sample population consisted of 8 digits, 18 letters, and the homographic "O." Some letters were not used because of their idiosyncratic shapes in the DEC character set. The ambiguous $1 / 1$ was excluded. Thus, those runs in which letters con- stituted the nontarget items (D/L and $\mathrm{L} / \mathrm{L}$ ) had more trials than those in which digits were nontargets (D/D and L/D). Although most experimenters choose a subset of the letter population to match the number of items in the digit sample, there were two reasons for not doing so here. First, restricting the letter population may produce artificial processing strategies. Second, no interactions between search condition and alphanumeric category have been previously reported (except Taylor, 1978, Experiment 2, when letter case was varied). No interactions would be expected here unless the previously used sampling constraint affected processing strategy.

Within each run in each condition, there were three types of nontarget item: (1) ordinary nontargets-These were either the digits 2-9 or the letters A B C D E F H J K N P R S T U V X Z. (2) Ambiguous nontargets-The alphanumeric character " $O$ " is ambiguous with respect to category membership; it was presented in each run. (3) Catch-trial nontarget-The catch-trial item was either (a) an item of the same category as the specified target item in the between-category condition, or (b) an item from a category that was different from that of the target and nontargets in the within-category condition. Gleitman and Jonides (1976, 1978) have shown that subjects tend to respond to a betweencategory catch-trial item as if it were the target item, if the item is presented late in the session after a series of between-category trials. In this experiment, a catch trial was presented in each run. RT, rather than response allocation, was the primary dependent variable: $R T$ is a much more sensitive metric with which to investigate underlying processing mechanisms (cf. Fletcher, 1981).

In those conditions in which letters were the nontarget items, a run of trials consisted of 48 ordinary nontargets $(3 \times 16), 1$ catch trial, 2 occurrences of the " $O$ " character, and 3, 4, or 5 targets. When digits were used as nontargets, 21 trials were presentations of ordinary nontargets, 1 catch trial, 2 “' $O$ " characters and either 3,4 , or 5 targets. All characters acted as target items. Although target characters could differ across subjects, any particular target was used in both the within- and between-category conditions in a session. Target and catch-trial items appeared in other runs as ordinary nontargets.

A session consisted of 24 runs, 6 of each condition. Thus, each subject received 996 trials in all, 96 of which were target trials. The order of the runs was randomized.

At the beginning of each run, the words "RESPOND 'TARGET' TO X, RESPOND 'NO' TO ALL OTHER ITEMS”' were presented on the CRT (' $X$ ' denoting the specified target character, changed for each run). The subject pressed a response key to signal readiness. The run was terminated by an "end of run" display.

\section{RESULTS AND DISCUSSION}

The mean correct RTs to target and nontarget characters, catch trials, and the homographic " $O$ " are shown in Table 1, which also presents the standard deviations for each mean. Error responses con-

Table 1

Mean Correct RTs (in Milliseconds) to Targets, Nontargets, Catch Trials, and “O” in Within- and Between-Category Conditions

\begin{tabular}{|c|c|c|c|c|c|c|c|c|}
\hline \multirow[b]{3}{*}{ Condition } & \multicolumn{4}{|c|}{ Digit Targets } & \multicolumn{4}{|c|}{ Letter Targets } \\
\hline & \multicolumn{2}{|c|}{ Within } & \multicolumn{2}{|c|}{ Between } & \multicolumn{2}{|c|}{ Within } & \multicolumn{2}{|c|}{ Between } \\
\hline & RT & $\mathrm{SD}$ & RT & $\mathrm{SD}$ & RT & SD & $\mathrm{RT}$ & SD \\
\hline Target & 630 & 192 & 594 & 159 & 573 & 123 & 545 & 121 \\
\hline Nontarget & 537 & 189 & 477 & 153 & 473 & 140 & 462 & 149 \\
\hline Catch Trial & 521 & 174 & 612 & 166 & 519 & 153 & 620 & 160 \\
\hline “O” & 530 & 164 & 507 & 148 & 456 & 118 & 485 & 140 \\
\hline
\end{tabular}


sisted almost exclusively of incorrect rejections of target items (6.5\% of target trials). There were no differences across conditions.

Separate analyses were performed on the RTs to ordinary target and nontarget stimuli, to catch trials, and to the " $O$ " character.

\section{Ordinary Target and Nontarget Trials}

The RT data were subjected to a four-way ANOVA - subjects (randomized) $\times$ search condition (withinor between-category) $\times$ target category (digit or letter) $\times$ response type (target or nontarget).

As predicted, the categorical relationship between the target and nontarget characters had a marked effect on processing time, revealed by the main effect of search condition $[F(1,8)=5.3, p<.05]$. Even with a display size of only one and a single target item, the items in the within-category condition took some $34 \mathrm{msec}$ longer to accept as target items (32 msec) or reject as nontarget items $(36 \mathrm{msec})$ than in the between-category condition. Search condition and response type did not interact $(\mathrm{F}<1)$.

RTs to target and nontarget items were larger with a digit target than with a letter target by some $46 \mathrm{msec}$. This target-category factor was significant $[F(1,8)=$ $6.6, p<.05$. Given that each mean in this comparison includes an equal number of conditions with letter and digit nontargets, it appears that digit targets took longer to search for than letters. Neither the interaction of target category and search condition $(F=1.1)$ nor the three-way interaction $(F=1.7)$ was significant.

Target RTs were almost $100 \mathrm{msec}$ larger than nontarget RTs $[F(1,8)=10.1, \mathrm{p}<.025]$.

It would appear, therefore, that the localization hypothesis, derived from multiple array studies to explain the differential performance in these two conditions, is not a sufficient explanation. Betweencategory processing benefit is present with a single array item. Moreover, the facilitation was observed on both target and nontarget trials, which supports the view that less processing of all items occurs in the between-category condition. This would be predicted by a parallel processes hypothesis (Taylor, 1978 ) in which categorization and identification are considered as parallel independent processes with stochastically overlapping termination times over trials. Both category and identity information is sufficient to discriminate targets from nontargets in the between-category condition, and the response can be executed upon the termination of either process, whichever is earliest. In the within-category condition, subjects can only utilize evidence from the identification process. Such a model may also predict faster explicit identification than categorization, if the mean of the termination times for the former process is presumed to be smaller.

\section{Catch Trials and Ordinary Nontargets}

It is clear from Table 1 that the catch-trial stimuli were processed differentially in the within- and between-category conditions. In the former, RTs to the catch trials (nontarget items from the otherwise unsampled category) were only some $15 \mathrm{msec}$ larger than response latencies to normal nontarget items. In the between-category conditions, on the other hand, the catch-trial stimuli (characters from the same category as the target item) took $147 \mathrm{msec}$ longer to classify than ordinary nontargets. A fourway ANOVA was performed on the response latencies to the catch trials and ordinary nontargets. Catchtrial RTs were significantly larger than ordinary nontarget RTs $[F(1,8)=11.7, \mathrm{p}<.01] ; \mathrm{RTs}$ to digit and letter nontargets did not differ significantly $(F<1)$, nor was there an overall benefit of the between- over the within-category search $[F(1,8)=2.2, p>.1]$. The only significant interaction was the marked one described above between search condition (between vs. within) and nontarget type (catch trials vs. ordinary nontargets) $[\mathrm{F}(1,8)=61.8, \mathrm{p}<.0005]$. Tukey's (a) test for unconfounded means on this interaction item produced a critical value of $34 \mathrm{msec}$ at the $5 \%$ level.

The pattern of results in the between-category condition is consistent with those reported by Gleitman and Jonides $(1976,1978)$. Although subjects did not respond to the catch trial as if it were a target item (i.e., by pressing the "TARGET" response key), it must be remembered that a catch-trial stimulus was presented in every run, not just on a single trial at the end of the session, as in Gleitman and Jonides' $(1976,1978)$ experiments. Nonetheless, it is clear that their RTs to these catch trials were markedly interfered with compared with normal nontarget processing. Having to make explicit the identity of the item slowed processing considerably. Catch-trial RTs in the within-category condition were not significantly different from ordinary nontarget RTs, as predicted.

\section{" $O$ " and Ordinary Nontargets}

The homographic character " $O$ " is ambiguous with respect to category membership. As such, categorical analysis of such items will not produce evidence to distinguish it from the specified target item. If early categorical processing can facilitate response latency when there is a categorical distinction between target and nontarget items, the facilitation observed in the between-category condition for ordinary nontargets should not be present for " $O$ " nontarget RTs. It can be seen from Table 1 that although ordinary nontarget RTs are smaller in the between-category condition, mean RT to the " $O$ ", character was not similarly facilitated. These RTs are not affected by search condition and do not differ significantly from those obtained to ordinary non- 
targets in the within-category condition. In a fourway ANOVA, this interaction between nontarget type and search condition was significant $[F(1,8)=$ $5.3, \mathrm{p}<.05$ ). The Tukey (a) test gave a critical value of $34 \mathrm{msec}(p<.05)$. Jonides and Gleitman (1972) report that the target " $O$ " can be treated as either a letter or digit, depending upon how it is defined. Clearly, when it occurs as a nontarget item, it shares a similar ambiguity which the instructional bias does not remove.

\section{GENERAL DISCUSSION}

A categorical distinction between the target and nontarget items facilitates both target acceptance and nontarget rejection latencies, even in a serial RT task in which localization cues cannot be utilized. Moreover, as indicated by the pattern of RT data obtained from the catch trials and the homographic "O," it appears that items in the between-category condition are not all processed to identity level.

Several points are worth consideration. These category effects were obtained with a single-array stimulus and target set of only one specified character. The marjority of the visual search studies reported utilized multiple target sets. It may be that multiple target sets encourage subjects to utilize category information when they would not normally do so (Karlin \& Bower, 1976). Corcoran and Jackson (1977), for example, suggest that partitioning stimuli by category is useful only when the subject expects to receive more different characters in the display than the number of possible categories.

If the localization hypothesis is inadequate to explain these data, within what framework can the effects be interpreted? Taylor's (1978) "horserace" model does provide one alternative (although Taylor implies that parallel processing occurs after target location). This stochastic model posits that the between-conditions advantage is due to the considerable degree of overlap between the identification and categorization distributions. The available literature, however, indicates that the mean RT difference between the distributions is quite large, on the order of $80-150 \mathrm{msec}$ (see the introduction). Unless the variance in the underlying distribution is very large, the degree of overlap will not be sufficient to predict the size of the reported category effects. The characteristics of the underlying distributions require careful empirical scrutiny before quantitative modelfitting is possible. Certain classes of parallel model, however, are ruled out by Taylor's (1978) finding that decision latencies based on either identity or category information are more variable than those based solely on identity information.

An alternative explanation of the category effects is to propose that they are produced at some stage after the initial encoding of the visual array (Gleitman \& Jonides, 1978)-the flatter slopes observed in between-category conditions reflects the reduced amount of time involved in target/array comparison. If it is assumed that the time to compare a representation of the target is a function of its similarity (or "code overlap") to the encoded information, the within-category condition will result in the largest RTs irrespective of whether or not the items are presented in a multiple array. This model does not rely on the use of location tags. Furthermore, "the question of whether identification codes are created before, after, or simultaneously with category codes is not relevant to the explanation of the category effect"' (Gleitman \& Jonides, 1978, p. 366). The model predicts that the more distractor items in an array (i.e., items from the same category as the target), the longer will be the comparison times, because there will be a greater similarity between the codes. In addition, because the spatial arrangement of distractors in a mixed-category array does not affect code overlap, grouping the distractors in the array (to localize search to a small portion of the array) should not facilitate RT. Finally, since similarity is equated here with functional entropy, repeating items in a multiple array should facilitate latencies in the within-category condition more than in the between-category condition.

\section{REFERENCES}

Atkinson, R. C., Holmgren, J. E., \& Juola, J. F. Processing time as influenced by the number of elements in a visual display. Perception \& Psychophysics, 1969, 6, 321-326.

Brand, J. Classification without identification in visual search. Quarterly Journal of Experimental Psychology, 1971, 23, 178186.

BUTLER, B. E. Selective attention and target search with brief visual displays. Quarterly Journal of Experimental Psychology, $1975,27,467-477$.

BUTLER, B. E. The category effect in visual search: Identification versus localisation factors. Canadian Journal of Psychology, 1980, 34, 238-247.

Corcoran, D. W. J., \& J JCKson, A. Basic processes and strategies in visual search. In S. Dornic (Ed.), Attention and performance $V I$. Hillsdale, N.J: Erlbaum, 1977.

Deutsch, J. A. On the category effect in visual search. Perception \& Psychophysics, 1977, 21, 590-592.

Dick, A. O. Processing time for naming and categorization of letters and numbers. Perception \& Psychophysics, 1971, 9, 350-352.

Egeth, H. E., Atkinson, J., Gilmore, G., \& Marcus, N. Factors affecting mode of processing in visual search. Perception \& Psychophysics, 1973, 13, 394-402.

Egeth, H. E., Jonides, J., \& WAll, S. Parallel processing of multi-element arrays. Cognitive Psychology, 1972, 3, 674-698.

Fletcher, B. (C). Wholistic and analytic stimulus processing: The development of selective perceptual strategies. Quarterly Journal of Experimental Psychology, 1981, 33A, 169-176.

Gleitman, H., \& Jonides, J. The cost of categorization in visual search: Incomplete processing of target and field items. Perception \& Psychophysics, 1976, 20, 281-288. 
Gleitman, H., \& Jonides, J. The effect of set on categorization in visual search. Perception \& Psychophysics, 1978, 24, 361-368.

INGLING, N. W. Categorization: A mechanism for rapid information processing. Journal of Experimental Psychology, 1972, 94, 239-243.

Jonides, J., \& Gleitman, H. A conceptual category effect in visual search: $\mathrm{O}$ as letter or as digit. Perception \& Psychophysics, 1972, 12, 457-460.

Jonides, J., \& Gleitman, H. The benefit of categorization in visual search: Target location without identification. Perception \& Psychophysics, 1976, 20, 289-298.

Karlin, M. M., \& Bower, G. H. Semantic effects in visual word search. Perception \& Psychophysics, 1976, 19, 417-424.

Ne isSE R, U. Decision time without reaction time: Experiments in visual search. American Journal of Psychology, 1963, 76, 376-385.

Sperling, G., Budiansky, J., Spivak, J. G., \& Johnson, M. C. Extremely rapid visual search: The maximum rate of scanning letters in the presence of a numeral. Science, 1971, 174, 33073311.

STERnBerg, S. Two operation in character recognition: Some evidence from reaction time measurements. Perception \& Psychophysics, 1967, 2, 45-53.

TAYLOR, D. A. Identification and categorization of letters and digits. Journal of Experimental Psychology: Human Perception and Performance, 1978, 4, 423-439.

White, M. J. Identification and categorization in visual search. Memory \& Cognition, 1977, 5, 648-657.

White, M. J. Naming and categorization of tilted alphanumeric characters do not require mental rotation. Bulletin of the Psychonomic Society, 1980, 15, 153-156.

(Manuscript received April 21, 1981; revision accepted for publication July 9, 1981.) 